\title{
La Cova de l'Avi (Vallirana, Barcelona) y el inicio del Neolítico final en el Nordeste de la Península Ibérica. Inhumaciones colectivas y nuevas redes de intercambio
}

\author{
La Cova de l'Avi (Vallirana, Barcelona) and the beginning of the Late Neolithic \\ in Northeastern Iberian Peninsula: collective inhnumations and new exchange networks
}

\author{
Joan Daura (*) \\ Montserrat Sanz (*) \\ F. Xavier Oms (**) \\ Mireia Pedro (**) \\ Pablo Martínez (**) \\ Ángel Rubio (***) \\ José-Miguel Tejero (****) \\ Xavier Mangado (**)
}

\author{
Jean Vaquer $(* * * * *)$ \\ Javier López-Cachero (**) \\ Mònica Oliva $(* * * * * *)$ \\ Antoni Asensio (*******) \\ Ramón Álvarez $(* * * * * * * *)$ \\ Josep Maria Fullola (**) \\ Maria Àngels Petit (**)
}

\section{RESUMEN}

Entre el Neolítico medio y el Neolítico final en el Nordeste de la Península Ibérica se producen cambios destaca-

(*) Centro de Arqueologia. Universidade de Lisboa (UNIARQ). Faculdade de Letras. Alameda da Universidade 1600-214 Lisboa, Portugal. Correos e: jdauralujan@campus. ul.pt; msanzborras@campus.ul.pt

(**) Seminari Estudis i Recerques Prehistòriques (SERP). Dept. Prehistòria, Historia Antiga i Arqueologia. Facultat de Geografia i Història. Universitat de Barcelona. C/ Montalegre 6-8.08001. Barcelona. Correos e.: xavieroms@gmail.com; mireiapedro@gmail.com; pablomartrod@gmail.com; mangado@ub.edu; xavierlopez@ub.edu; fullola@ub.edu; petit@ub.edu

(***) Laboratorio de antropología física. Facultad de Medicina. Universidad de Granada. Av. de Madrid 11. 18012 Granada. Correo e: a_rubiosalvador@hotmail.com

(****) CNRS UMR 7041 ArScAn Equipe Ethnologie préhistorique. 21 Allée de l'Université 92023. Nanterre CEDEX. France. Correo e: jose-miguel.tejero@mae.cnrs.fr

(*****) Laboratoire Travaux et Recherches Archéologiques sur les Cultures, les Espaces et les Sociétés (TRACES). Université de Toulouse 2 1- Jean Jaurès, Maison de la Recherche. 5 allée Antonio-Machado. F-31058 Toulouse cedex 9.

Correo e.: jean-sebastien.vaquer@orange.fr

$(* * * * * *)$ Dept. de Prehistòria, Universitat Autònoma de Barcelona. Edifici B, Facultat de Filosofia i Lletres. 08193 Bellaterra. Correo e.: monicaolivapoveda@gmail.com dos en las pautas funerarias y en la cultura material de los grupos humanos, especialmente en los ajuares y lugares de enterramiento. Durante el Neolítico medio-reciente son típicas las inhumaciones primarias individuales en fosas con ajuares de cuentas de variscita, sílex melado, vasos cerámicos y puntualmente obsidiana. Por el contrario, durante el Neolítico final se generalizan las inhumaciones colectivas y sucesivas en cuevas y abrigos con ajuares que se caracterizan por la gran disminución de la variscita, sustituida por una gran variedad de colgantes y cuentas en piedra, concha y hueso. Ahora también aparecen las grandes láminas y los puñales de sílex, en ocasiones de procedencia extrapeninsular. La Cova de l'Avi representa en la actualidad el yacimiento cronológicamente más antiguo (c. $4700 \mathrm{BP}$ ) en el que se documentan estos nuevos comportamientos funerarios.

\section{ABSTRACT}

The Middle to Late Neolithic transition in NE of Iberian Peninsula is characterized by changes in mortuary practices and grave goods. In the Middle Neolithic single

(*******) Secció Espeleològica de l'Ordal (SEO)-Centre Excursionista de Vallirana (CEV). C/ Major 402. 08759 Vallirana. Barcelona. Correo e.: tasensiov@gmail.com

$(* * * * * * *)$ Dept. Prehistòria, Historia Antiga i Arqueologia. Facultat de Geografia i Història. Universitat de Barcelona. C/ Montalegre 6-8. 08001 Barcelona. Correo e: ralvarez@ub.edu Recibido 3-XI-2014; aceptado 16-II-2105. 
primary burials are associated with grave goods of variscite beads, honey-colored flint blades and occasionally obsidian. During the Late Neolithic these are replaced for collective inhumations in cave and rock-shelters with grave goods composed mainly by shell, bone and stone beads and pendants, large flint blades and flint daggers, some from outside the Iberian Peninsula. Cova de l'Avi represents the earliest site (c. $4700 \mathrm{BP}$ ) at which these changes in mortuary practice have been documented.

Palabras clave: Neolítico medio-reciente; Neolítico final; Nordeste Península Ibérica; Prácticas funerarias; Intercambios a larga distancia; Materias primas líticas; Puñal tipo Châtaigniers.

Key words: Late-Middle Neolithic; Late Neolithic; Northeastern Iberian Peninsula; Burial ritual; Long distance exchange; Lithic Raw Materials; Flint dagger.

\section{INTRODUCCIÓN}

El Neolítico medio-reciente en el Nordeste de la Península Ibérica (c. 4200-3400 cal BC) se caracteriza por una marcada regionalización, visible especialmente en las pautas funerarias. La zona que ocupa la Depresión Prelitoral Catalana (VallésPenedés) se ha definido básicamente por sepulturas individuales en fosas, las cuales llegan a formar grandes necrópolis (Gibaja 2003; Roig et al. 2010). Esta zona se corresponde con el grupo denominado Vallesiense, que se enmarca en un engranaje cultural de intercambios y relaciones a largas distancias (Martín y Villalba 1999; Gibaja 2003) donde intervienen otros territorios y grupos, tales como el Chassey francés y eventualmente el Cortaillod suizo y el Vaso a Bocca Quadrata italiano.

Los ajuares cuentan con sílex melado del sureste francés (Vaquer 2012), obsidiana de la isla de Cerdeña (Gibaja et al. 2014; Terradas et al. 2014), rocas de tipo alpino (Vaquer et al. 2012) y vasos de boca cuadrada de filiación norte o centroitaliana (Bosch y Gómez 2009). Las cuentas de variscita, procedente de Can Tintorer (Villalba et al. 2011), también son habituales. Por motivos que aún se desconocen, este sistema y sus redes se colapsan y desaparecen a partir de 3600-3400 cal BC, dando lugar a un Neolítico final (c. 3500 - 2300 cal BC) caracterizado por cambios sustanciales en aspectos económicos, de hábitat y también en las pautas funerarias (Martín 2003). Se generalizan los sepulcros megalíticos, los hi- pogeos y el uso de las cavidades naturales como sepulcros colectivos. Algunos de los elementos que habían marcado culturalmente los ajuares hasta ese momento desaparecen por completo, otros disminuyen y son sustituidos por otros nuevos indicativos de nuevas redes de intercambio (Vaquer 2012). En el Neolítico final se documentan, entre otras, grandes láminas de sílex de la cuenca de Apt-Forcalquier (Alta Provenza, Francia) e influencias cerámicas del sudeste francés, de los grupos Treilles y Ferrières, Saint-Pons y en menor medida Fontbouïsse. De igual modo, surge todo un conjunto de colgantes y cuentas confeccionadas a partir de una gran variedad de conchas marinas, minerales, rocas y hueso (Petit y LópezCachero 2011; Cebrià et al. 2013).

La Cova de l'Avi, por su cronología, es uno de los primeros yacimientos donde se documenta este cambio de pautas funerarias en el nordeste peninsular, especialmente en lo que se refiere a (i) lugar de enterramiento, (ii) tipo de sepultura y (iii) ajuar. Resulta llamativo que cuando la Cova de l'Avi se utiliza como lugar de inhumación, todavía existen enterramientos que siguen las pautas anteriores (Martí et al. 1997; Roig et al. 2010).

\section{LA COVA DE L'AVI}

La Cova de l'Avi (41'22'47.80'N 1'53'38.69'"E, 523 m s.n.m.), conocida también como Cova de la Vall o de la Moneda (Asensio 2001; Clua et al. 2013) está situada en el macizo del Garraf, $20 \mathrm{~km}$ al NO de Barcelona (Fig. 1.1 a 1.3). La cueva se desarrolla sobre una grieta producida por la fractura de las calizas así como por la descompresión y el retroceso del escarpe. Las aguas meteóricas han utilizado este conducto como canal preferente y han modelado la disposición alargada de la cavidad. El acceso se encuentra $9 \mathrm{~m}$ por encima de la base del escarpe y requiere la utilización de medios espeleológicos (Fig. 1.7 y 1.8). Tras flanquear un resalte $(\mathrm{R})$ ascendente se pasa a la galería principal (GP) (Fig. 1.8 y 1.9), relativamente estrecha, que desemboca en una sala más espaciosa (S) de planta circular y sin luz directa (Fig. 1.10).

Los miembros del Centre Excursionista de Vallirana (CEV) la descubrieron en los 1960, excavando la GP y S. La cavidad permaneció prácticamente inédita hasta nuevos estudios es-

Trab. Prehist., 72, N. ${ }^{\circ}$ 2, julio-diciembre 2015, pp. 327-341, ISSN: 0082-5638

doi: $10.3989 /$ tp. 2015.12157 

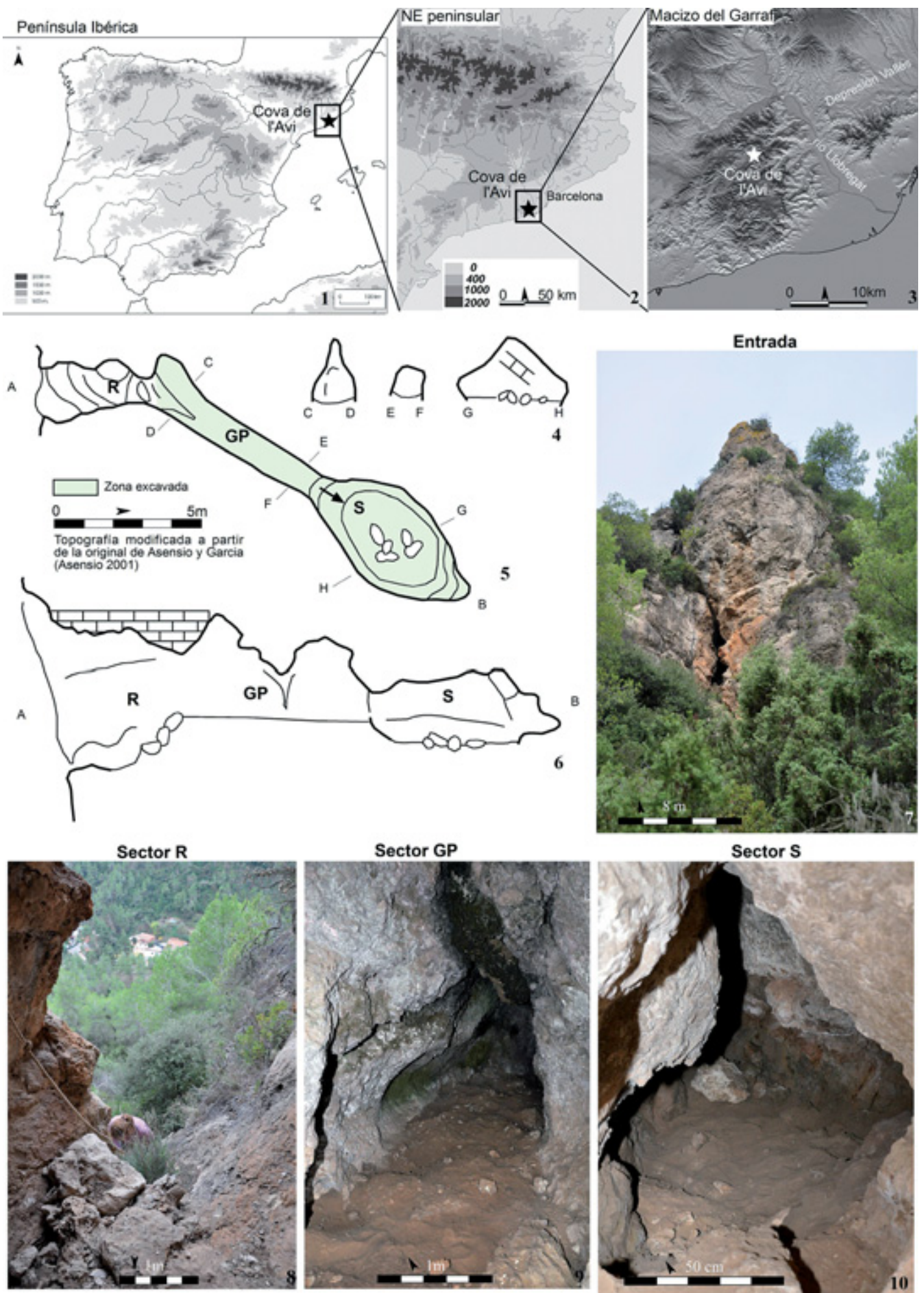

Fig. 1. Cova de l'Avi (Vallirana, Barcelona). 1-3: Situación. 4: Secciones transversales. 5: Planta con el área excavada en la intervención del Centre Excursionista de Vallirana. 6: Sección longitudinal. 7: Cavidad vista desde el exterior. 8: Acceso a la cavidad y sector R. 9: Vista de la Galería Principal (GP) desde la entrada. 10: Vista de la S desde la GP. 
peleológicos (Asensio 2001). En 2012 se tamizó la escombrera de los años 1960. En el presente trabajo se estudian los materiales arqueológicos procedentes de ambas colecciones.

\section{RESTOS ÓSEOS HUMANOS Y CRONOLOGÍA}

Los restos óseos humanos ascienden a 667, presentan un deficiente estado de conservación y un tamaño reducido. La metodología empleada en su estudio ha seguido procedimientos estándares. La edad se ha determinado a partir de la erupción de las piezas dentarias, del grado de sinostosis de las suturas craneales y de los cambios en la sínfisis púbica, mientras que, para el sexo, se han considerado los caracteres morfológicos del cráneo y de la pelvis, así como funciones discriminantes. Se ha establecido un NMI de 12: 5 subadultos y 7 adultos (4 masculinos y 3 femeninos). En líneas generales, las características de esta población son similares a las de los grupos humanos del Neolíti- co del nordeste peninsular (Gibaja et al. 2010). La población es joven, con un $41,6 \%$ de mortalidad infantil. Sólo un adulto supera los 40 años. El dimorfismo sexual está muy acentuado y es diferenciable visualmente y cuantificable métricamente. Los individuos masculinos son más robustos con gran desarrollo de las entesas musculares. La repetición de caracteres epigenéticos observable en el esqueleto podría sugerir una posible relación de parentesco entre los individuos.

En cuanto a la salud y la enfermedad el gran desgaste en los dientes, sobre todo en los molares, indicaría una dieta muy abrasiva. De las 262 piezas analizadas un 2,6\% tienen caries, una frecuencia inferior a la mayoría de las series neolíticas de nordeste peninsular (Subirà et al. 2014). Uno de los individuos presenta enfermedad periodontal con un retroceso alveolar provocado por la inflamación de la encía. Esta patología puede estar causada por bacterias, cálculos, enclavamiento de alimentos $\mathrm{u}$ otros agentes externos. Como enfermedades osteoarticulares se ha detectado artrosis en las vértebras lumbares y también en las articulaciones inferiores de dos individuos masculinos.

\begin{tabular}{|c|c|c|c|c|c|c|c|c|}
\hline \# Individ. & Sexo & $\begin{array}{c}\text { Grupo } \\
\text { de edad }\end{array}$ & \# Inv. & Parte & Código Lab. & $\delta 13$ & Edad BP & cal BC 2 sigmas \\
\hline 1 & - & Infantil I & & & & & & \\
\hline 2 & - & Infantil I & & & & & & \\
\hline 3 & - & Infantil II & & & & & & \\
\hline 4 & - & Infantil II & & & & & & \\
\hline 5 & q & Juvenil & & & & & & \\
\hline 6 & q & Maduro & & & & & & \\
\hline 7 & $0^{\lambda}$ & Adulto & $\begin{array}{l}\text { CDA- } \\
21-2\end{array}$ & PM2 & OxA-29611 & $-20,82$ & $4696 \pm 30$ & $\begin{array}{l}3629-3584(13,9 \%) \\
3531-3487(21,8 \%) \\
3474-3372(59,7 \%)\end{array}$ \\
\hline 8 & $0^{\lambda}$ & Adulto & $\begin{array}{l}\text { CDA- } \\
20-2\end{array}$ & M2 & OxA-29610 & -19.20 & $4703 \pm 32$ & $\begin{array}{rr}3631-3579 & (19 \%) \\
3535-3488 & (21,7 \%) \\
3472-3372 & (54,4 \%) \\
\end{array}$ \\
\hline 9 & $0^{\pi}$ & Adulto & & & & & & \\
\hline 10 & $0^{\pi}$ & Adulto & & & & & & \\
\hline 11 & q & Adulto & & & & & & \\
\hline 12 & q & Adulto & & & & & & \\
\hline
\end{tabular}

Tab. 1. Cova de l'Avi (Vallirana, Barcelona): individuos, sexo, grupo de edad y dataciones radiocarbónicas de los restos óseos humanos. Dataciones calibradas con OXCAL 4.2 (acceso 06-V-2014) en base a la curva Intcal13 (Reiner et al. 2013).

Trab. Prehist., 72, N. ${ }^{\circ}$ 2, julio-diciembre 2015, pp. 327-341, ISSN: 0082-5638

doi: $10.3989 /$ tp. 2015.12157 
De etiología metabólica se han identificado bandas de hipoplasia en el 7,7\% de las piezas dentarias, frecuencia inferior a la de otros contextos contemporáneos (Subirà et al. 2014). Al menos 4 individuos de la población sufrieron estos episodios entre el primer y el segundo año de vida (Goodman y Rose 1990), posiblemente, provocados por deficiencias nutricionales causadas por el destete. Se ha reconocido un caso de periostitis (engrosamiento) en la parte posterior de la epífisis distal de una fíbula, cuyo origen podría estar relacionado con alguna infección sistémica, traumas, tumores o procesos vasculares. Por último, una mujer madura presenta un pequeño traumatismo ante mortem que afectó a la tabla externa y al diploe en la parte derecha del frontal. En el Neolítico, el uso de armas contundentes, cortantes y punzantes deja lesiones identificables en los restos esqueléticos registradas en otras series neolíticas (Vegas 2007).

La cronología del yacimiento se basa en dos dataciones de ${ }^{14} \mathrm{C}$ sobre dos dientes humanos extraídos de dos mandíbulas diferenciadas, realizadas en la Oxford Radiocarbon Accelerator Unit (ORAU). Se presentan calibradas (Tab. 1) en base a la curva INTCAL13 (Reimer et al. 2013). Una muestra, atribuida al individuo 8 , presenta un ran-

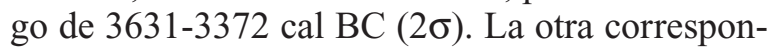
de al 7 y su rango es de 3629-3372 cal BC $(2 \sigma)$. Ambas se sitúan a mediados del IV milenio cal $\mathrm{BC}$ y son estadísticamente iguales.

\section{MATERIALES ARQUEOLÓGICOS}

Los materiales arqueológicos comprenden punzones óseos, ornamentos de piedra y hueso, cerámica e industria de sílex.

La industria ósea se ha analizado tipo-tecnológicamente y estudiado mediante microscopio estereoscópico modelo Olympus SZ61 (entre 10x y $45 \mathrm{x}$ ). Comprende 4 punzones, uno en asta de corzo (Fig. 2.4) y tres en hueso de ovicaprino (metápodo Fig. 2.3; tibias Fig. 2.1 y 2.2). Este último soporte es de uso recurrente durante toda la Prehistoria reciente (Camps Fabrer et al. 1990), mientras que el asta no es tan habitual (Llobera 1986; Camps Fabrer y Ramseyer 1990).

Todos los punzones reflejan las mismas concepciones técnicas. En los soportes en hueso se ha fracturado la diáfisis por percusión directa junto a las epífisis, reservando las epífisis distales como zonas de prensión. Esta intervención comporta un plano de fractura oblicuo en relación al eje longitudinal del hueso regularizado en parte durante la fase de confección del objeto. La extensión del raspado se limita a la zona medio-distal que permite apuntar la parte activa del útil. Desde el punto de vista funcional su aplicación no resulta imprescindible y puede responder tanto a una regularización más cuidadosa, como a una elección cultural.

El punzón en asta (Fig. 2.4) muestra dos fracturas en lengüeta a la altura de una de las puntas de la corona y de la percha. El plano de fractura está asociado a un arrancamiento en flexión, aunque la secuencia técnica completa no es identificable. Carece de acondicionamiento ya que su configuración natural hace innecesario modificar el soporte.

En relación al uso, los soportes en hueso presentan en la zona distal un lustre asociado a estrías, sin embargo es complicado diferenciar entre sí las funcionales, tecnológicas y de reavivado. En las fracturas distales (dos en lengüeta y una en dientes de sierra) son frecuentes las originadas por presión. En el punzón de asta la serie de incisiones oblicuas en la zona distal asociada a lustre puede haberse producido tanto por procesos de trabajo por perforación como por el propio animal.

Los ornamentos recuperados son 7. Uno es un incisivo inferior de suido (Sus sp.) (Fig. 2.5) perforado para acondicionar un sistema de suspensión tipo colgante de gargantilla (Ambert 2003). Tras el pulido de la pieza se encajan 8 ranuras semiperimetrales a lo largo de la raíz (Fig. 2.5b). La morfología de las estrías indica que con toda probabilidad se ejecutó por frotación repetida con un cordel empleando posiblemente arena como material abrasivo. La perforación está fracturada de antiguo. Es circular, de sección bicónica y se localiza en la zona oclusal (Fig. 2.5a) que, previamente, se adelgazó por abrasión. Como sucede en muchas de estas piezas dentarias, la perforación se efectuó por rotación continua bifacial con arco. El segundo colgante (Fig. 2.6) está realizado sobre un fragmento de concha de un gran gasterópodo (Strombus bubonius o Charonia sp.). Tiene dos perforaciones unifaciales ejecutadas desde la cara superior del objeto. Por encima de ellas se observa una depresión correspondiente a sendas perforaciones fracturadas de antiguo. En la cara superior 

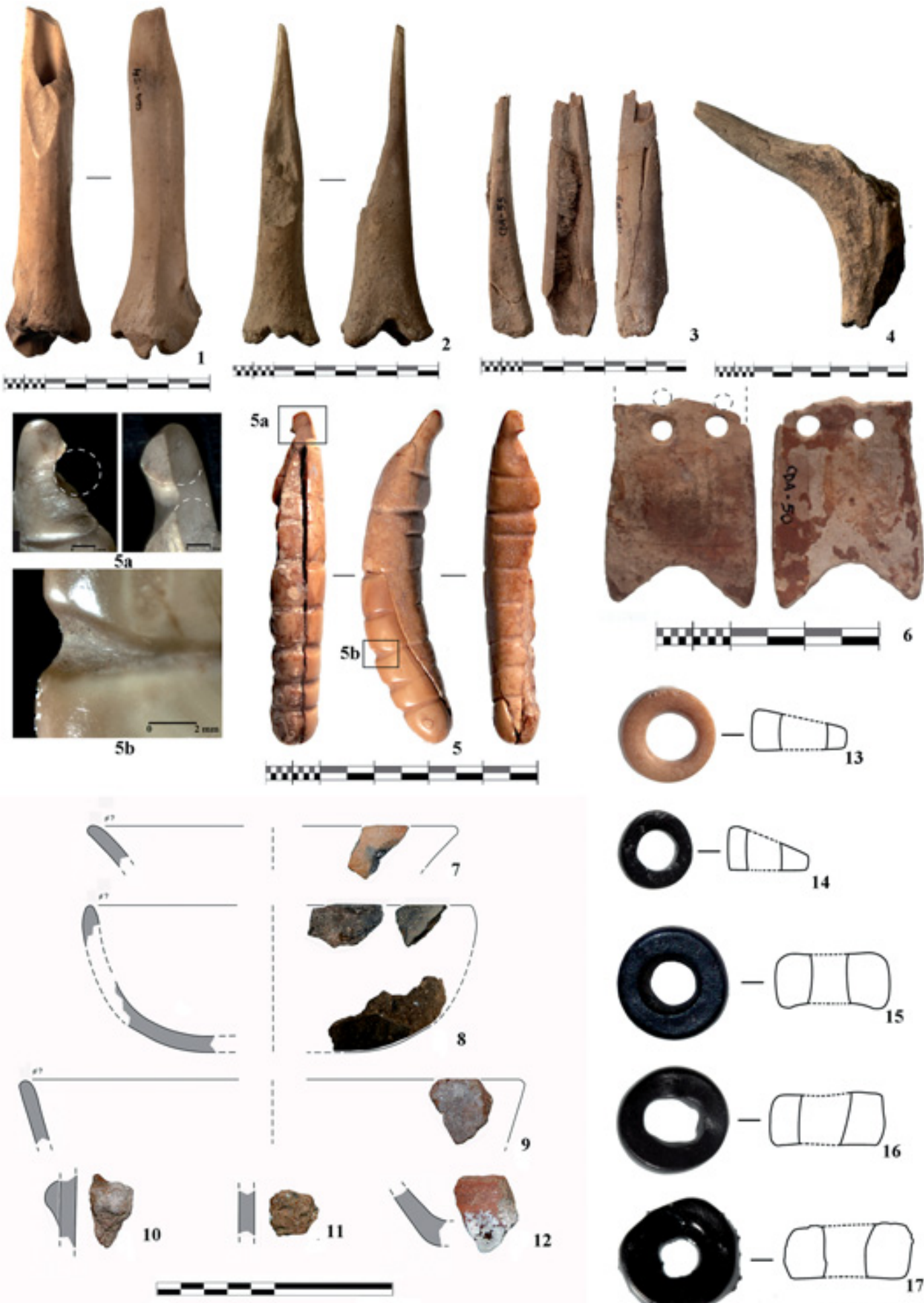

2

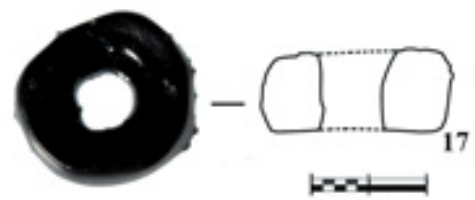

Fig. 2. Industria ósea. Punzones: 1 y 2. Tibia de Ovis/Capra, 3. Metápodo de ovicaprino, 4. Punta de corona de asta de corzo. Colgantes: 5. Diente de suido, 6. Concha de gasterópodo. Cerámica: 7-9 y 12. Formas. 10-11. Fragmentos decorados. Cuentas de collar: 13. Concha, 14-17. Enstatita.

Trab. Prehist., 72, N. ${ }^{\circ}$ 2, julio-diciembre 2015, pp. 327-341, ISSN: 0082-5638

doi: $10.3989 /$ tp.2015.12157 
se disponen de forma transversal una serie de incisiones muy finas presentes longitudinalmente en la cara inferior. Los orificios son circulares y parecen ejecutados por rotación continua posiblemente con empleo de arco. La morfología bífida de la parte inferior sugiere que el artesano recortó así la concha para cambiar parte de su morfología natural.

Además hay 5 cuentas de collar con perforación central bipolar: 4 minerales y 1 sobre fragmento de concha. Se han analizado según la metodología de Oliva $(2004,2010)$ y observado con lupa binocular Leica A16 (20x - 80x). La cuenta discoidal de concha es de la familia de las cardidae (Cardium sp) (Fig. 2.13). Las minerales (Fig. 2.14-17) son de color negro y forma discoidal-circular. El empleo de un difractograma de rayos-X (DRX) (Fig. 2.14) permitió identificar la materia prima de una de ellas como enstatita. Es un piroxeno de silicato de magnesio de dureza 5-6 asociado a rocas magmáticas, como las basálticas y otro tipo de plutónicas (Roscian et al. 1992) aunque algunos autores proponen que puedan ser el resultado de una manufactura intencional a partir de esteatita (Panei et al. 2005).

Las trazas tecnológicas muestran un proceso de abrasión multidireccional que, en la cuenta de concha, se hizo contra una superficie de grano fino $\mathrm{y}$ en las de mineral, contra una piedra de grano grueso que no consiguió erradicar irregularidades de la materia prima. En todas las cuentas se ven las estrías concéntricas del orificio central de suspensión, producto de la perforación con una punta lítica en un soporte a modo de trépano giratorio rotativo (volante de inercia). El acabado bruñido de la concha se hizo, posiblemente, contra una materia tipo cuero. El lustre por fregamiento diferencial reiterado en zonas de la superficie cercana al contorno se debe a que las cuentas debían ir suspendidas o ensartadas en un cordel o tendón.

El conjunto cerámico comprende 14 restos: 4 bordes (dos de un mismo vaso), 2 bases y 8 informes que pertenecen a tres vasos distintos. El primero es de borde secante e inclinado hacia el exterior con labio oblicuo al interior y acabado alisado (Fig. 2.7). El segundo es un cuenco hemisférico bruñido con borde secante e inclinado al exterior, labio adelgazado, y base cóncava (Fig. 2.8). El tercero tiene un borde secante algo inclinado al exterior de perfil rectilíneo, labio redondeado y acabado rugoso (Fig. 2.9). Dos fragmentos informes de acabado rugoso están decorados con un cordón impreso digitado, dispuesto en horizontal (Fig. 2.10), y con impresiones unguladas (Fig. 2.11). Una de las bases es plana y con acabado tendente al bruñido (Fig. 2.12).

La elevada fragmentación y escasez de restos del conjunto dificulta su contextualización. El único perfil completo restituible es un cuenco que puede considerarse típico de ciertos contextos funerarios del Neolítico. No obstante las decoraciones descritas son ajenas a un contexto de la segunda mitad del IV milenio, en el que tampoco son frecuentes las bases planas ni los bordes muy exvasados (Fig. 2.7). Todos estos elementos son más propios de la Edad del Bronce inicial e incluso las decoraciones unguladas podrían remontarse a una etapa evolucionada de la misma. En general, es habitual la escasez de vasos cerámicos asociados a conjuntos funerarios del Neolítico Final precampaniforme en la zona del Garraf-Penedés (Petit y López Cachero 2011) y por extensión en el Nordeste peninsular. En caso de haberlos, los cuencos suelen ser una de las formas habituales.

El conjunto lítico está compuesto por 4 grandes láminas, 5 puntas de flecha y 1 puñal. Las puntas se han clasificado según Cabanilles (2008) y el puñal según Vaquer et al. (2014). Las láminas se han descrito morfológica y tecnológicamente. El sílex se ha observado siempre a grandes aumentos $(\leq 40 x)$.

Una lámina (Fig. 3.9) está realizada en sílex continental-lacustre con vestigios de tallos de algas del género Chara (Fig. 3.9a) atrapadas en el gel de sílice. Estas características macroscópicas son las del denominado sílex de la cuenca de Apt-Forcalquier (Francia). La segunda (Fig. 3.6) presenta bioclásticos de ostrácodos y probables secciones de tallos de algas carofíceas. Los parámetros técnicos indican su obtención mediante percusión indirecta a partir de un núcleo configurado de manera frontal bidireccional. La tercera (Fig. 3.8) está configurada en un sílex blanquecino, azoico y traslúcido de origen indeterminable que muestra tratamiento térmico (lustre cerúleo). Se observan algunos desgastes y rastros de reavivado. La cuarta (Fig. 3.7) está fragmentada en cuatro y completamente patinada. Su estado de conservación no permite ninguna aproximación.

La materia prima de todas las puntas de flecha corresponde a materiales silíceos propios de cuencas evaporíticas. En todas se observa lustre céreo, característico del tratamiento térmico tecnológico. 

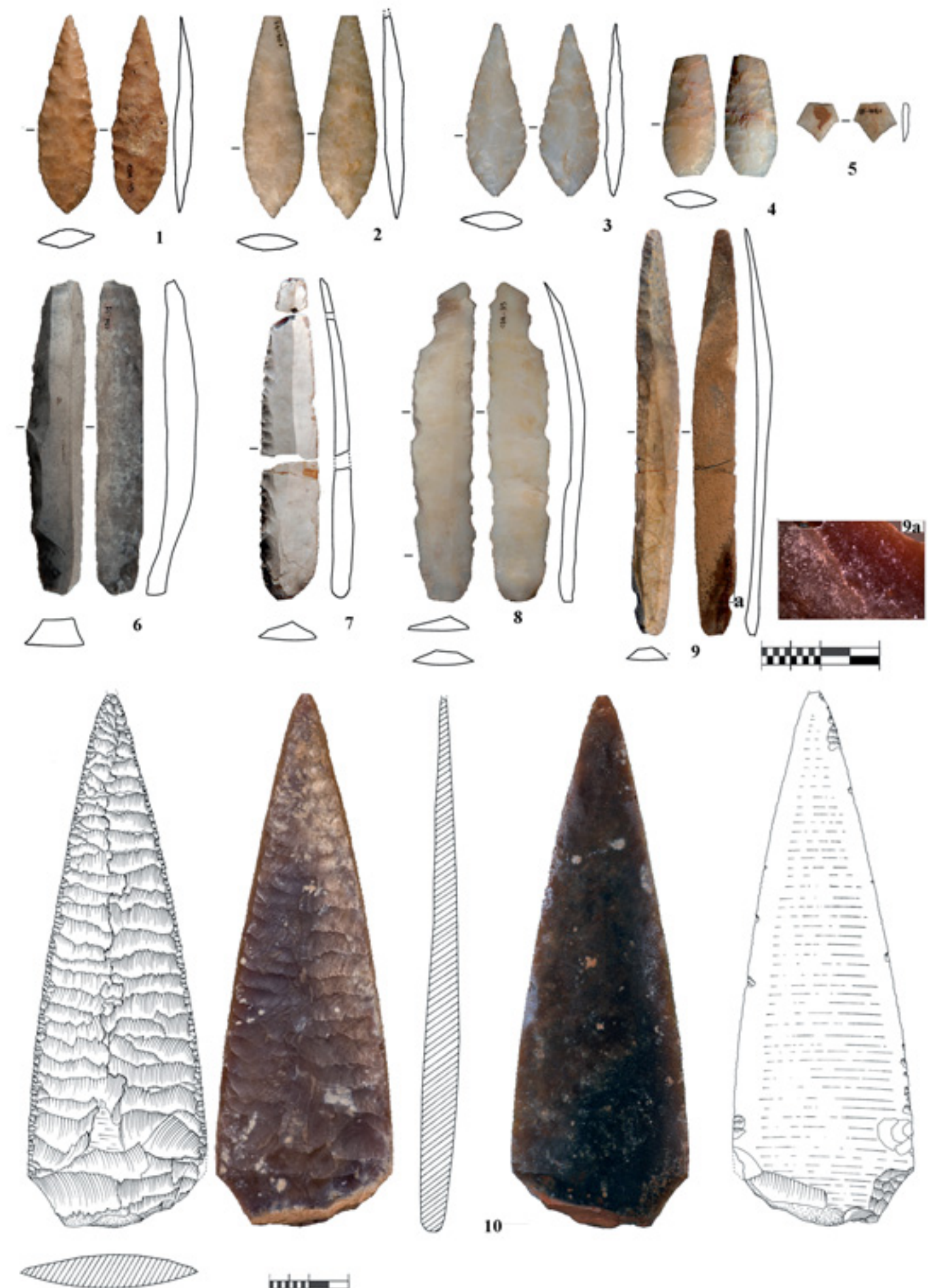

\section{mine=}

Fig. 3. Industria de sílex. 1-5: Puntas de flecha. 6-9: Grandes láminas. 9a: anillo Liesegang. 10: Hoja de puñal tipo Châtaigniers en sílex de Apt-Forcalquier (dibujo J. Vaquer).

Trab. Prehist., 72, N. ${ }^{\circ}$ 2, julio-diciembre 2015, pp. 327-341, ISSN: 0082-5638

doi: $10.3989 /$ tp.2015.12157 
Tres (Fig. 3.1-3) son puntas foliformes simétricas de base apuntada, tipo PF5 de Cabanilles (2008). La cuarta (Fig. 3.4) es foliácea pero de morfología losángica. La quinta conserva solo un pedúnculo y aletas incipientes (Fig. 3.5).

El puñal de sílex es la pieza más significativa del yacimiento. Es de tipo Châtaigniers (Vaquer et al. 2014) con retoque dorsal en bufanda y dorso ventral pulido (Fig. 3.10).

Todas las características observadas a la lupa binocular, concuerdan con la definición macroscópica de los sílex oligocenos del valle del Largue, y de manera más precisa aún, con las facies de la zona de Pary, en Saint-Michel-l'Observatoire, en los Alpes de Alta Provenza, que pertenecen a los de la cuenca oligocena de Apt-Forcalquier (Renault y Bressy 2007).

La cadena operativa implica la talla de la preforma sobre un fragmento de hoja gruesa. Después se lleva a cabo el pulido integral de ambas caras del soporte, salvo una pequeña parte cortical en la base de la lengüeta. Considerando la longitud de la pieza, es probablemente un relicto cortical de la parte distal del soporte. Las hojas con córtex distal son frecuentes en los contextos provenzales del Neolítico final y del Calcolítico. Durante el pulido se ha invertido el sentido de la pieza en relación al eje de la talla, para que la punta del puñal sea más gruesa que la base. Seguidamente, la cara dorsal, más convexa, ha sido retocada por presión, de manera que cada nuevo levantamiento recortara parte del anterior. El retoque en bufanda ha progresado en sentido horario, como se observa por el recorte de las respectivas lancettes. Esta misma progresión se ha documentado en otros puñales de retoque en bufanda en sílex de Forcalquier del Mediodía francés (Vaquer et al. 2014). Seguidamente los filos se regularizaron de modo marginal y oblicuo con un compresor fino creando pequeñas denticulaciones.

\section{DISCUSIÓN}

Los materiales de la Cova de l'Avi son homogéneos pero debe tenerse en cuenta antes de cualquier conclusión que perdieron su contexto de procedencia y que la representatividad de las dos fechas obtenidas a partir de un par de individuos es limitada, ya que eran doce en total. Ello no impide que la antigüedad de las mismas (c. 4700 BP) de pie a reflexionar sobre su significación en el contexto del Neolítico final precampaniforme.

Son pocos los yacimientos considerados como Neolítico final tipo veraciense o Neolítico final precampaniforme que dispongan de fechas tan antiguas en el nordeste de la Península Ibérica. Las dataciones en la mayoría de ellos son de baja fiabilidad debido a que las muestras combinan varios carbones de un mismo nivel, como en el Coll y la Font del Molinot, o distintos huesos humanos en la Cova del Toixó (Martín y Mestres 2002) (Fig. 4). Yacimientos con muestras únicas, como la Cova de les Agulles (Gómez et al. 2011) o la Cova del Pantà de Foix (Cebrià et al. 2013), nos remiten asimismo a un IV milenio algo más avanzado. Ambos registros y la Cova de l'Avi serían indicativos de que, en el nordeste peninsular, ya hay contextos propios del Neolítico final en un momento de disolución del Neolítico medio-reciente (Vallesiense), fase transicional que algunos autores han denominado como Neolítico reciente (Martín y Villalba 1999; Martín y Mestres 2002; Martín 2003). Es decir, mientras en la Cova de l'Avi se realizarían enterramientos colectivos todavía, la perduración de sepulturas individuales y dobles estaría atestiguada en las necrópolis de Can Gambús y de Bòbila Madurell (Martí et al. 1997: Roig et al. 2010) en torno a 4700-4500 BP.

El conjunto material de la Cova de l'Avi, de ser cronológicamente homogéneo, podría caracterizar este momento transicional entre el Neolítico medio-reciente y el final. Ello nos daría pie a reflexionar sobre la significación de la cultura material en base a la analogía con registros arqueológicos similares.

El conjunto cerámico resulta prácticamente banal, salvo el cuenco hemisférico bruñido que podría formar parte del ajuar funerario por lo que conocemos en otros yacimientos sepulcrales de la región (Petit 2001; Cebrià et al. 2013). En cambio el resto de las formas y decoraciones cerámicas se sitúan en un horizonte cronológico más avanzado.

El utillaje lítico, especialmente las grandes láminas y las puntas de flecha, así como las cuentas de collar y los punzones óseos se integran bien en los conjuntos funerarios colectivos del Neolítico final. Alguna de las láminas presenta posibles reavivados, quizás, de uso. Sus materias primas 


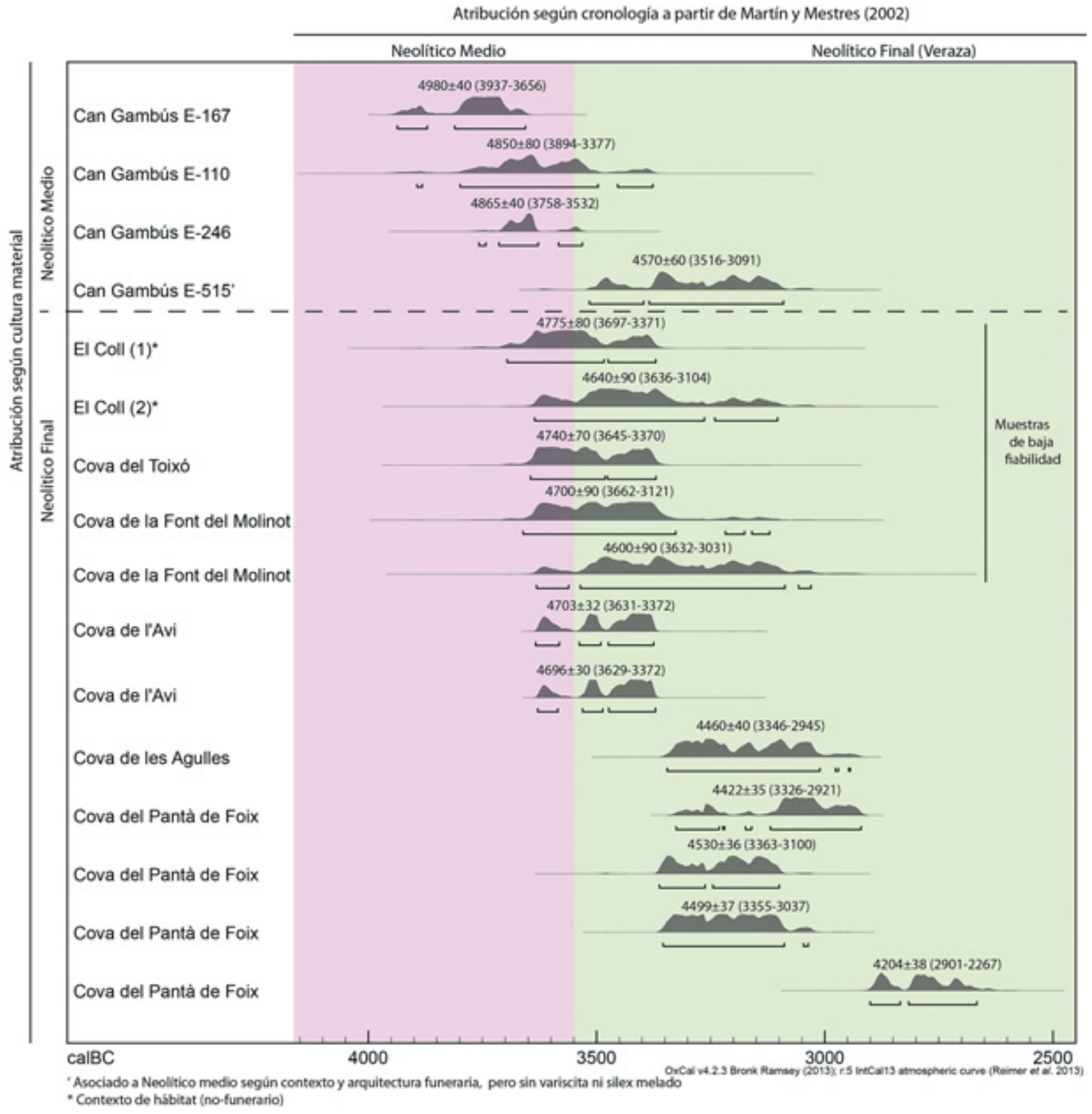

Fig. 4. Gráfico múltiple con las principales dataciones mencionadas en el texto. Edades radiocarbónicas BP y edades calibradas AC entre paréntesis mediante OxCal 4.3 (Bronk Ramsey 2013) en base a la curva Intcal13 (Reimer et al. 2013 ).

tienen varias procedencias. El sílex lacustre de una de las láminas podría relacionarse con la cuenca sedimentaria endorreica surpirenaica. En cambio hay otros dos sílex extrarregionales con afloramientos localizados en la cuenca de AptForcalquier. La presencia de éstos últimos ma- teriales en el Nordeste peninsular no es extraña, pero está poco documentada (Vaquer 2012).

La tipología de las puntas de flecha (foliáceas, menos una con pedúnculo y aletas incipientes) de la Cova de l'Avi no es diagnóstica desde el punto de vista cronológico en el nordeste penin- 


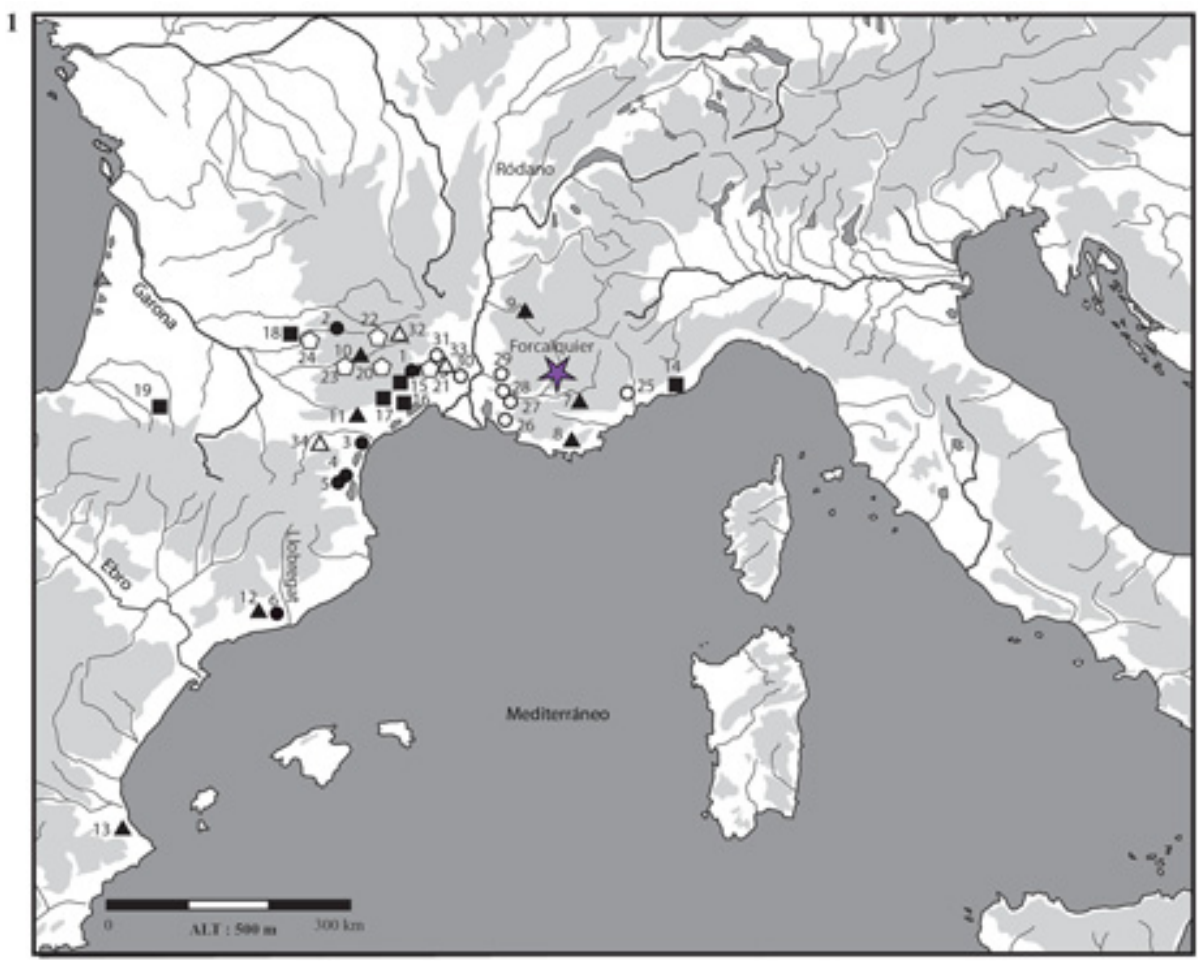

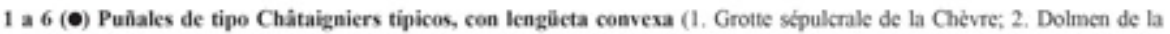
Gachette; 3. Grotte sípulerale de Bringairet; 4. Grotte des Châtaigniers; 5. Grotie de l'Amaga de la Dona; 6. Cova de 1'Avi). 7 a 13 (A): Puñales de tipo Font de Molinot, con lengũeta trianzular (7, Dolmen du Roque d'Aille; 8. Dolmen de Gacutabry; 9 . Grotie du Foumet; 10. Dolmen de la Blaquière: 11. Dolmen de Jappeloup; 12. Cova de la Font del Molisot; 13. Cova de I'Infern). 14 a 19 (ש) Punales tipo Taillan, con lengūeta traperoidal (14. Caverna del Torello; 15. Grotte du Salpétre; 16. Tumulus de Pech Rouguier, 17. Dolmen du Bois de Marou; 18. Dolmen de Peyrolevado; 19. Tumulus B de Taillan). 20 a 24 (O) Tipos recertados o modificades (20. Dolmen du Planas; 21. Dolmen des Raseassols; 22. Dolmen 2 de La Blachère; 23. Grotte 2 des Cascades; 24. Chiteau de la Prune), 25 a 32 ( $\Delta$ ) Ejemplos fragmentados o perdidos (25. Tumulus de Colette; 26. Tombe d'Enco de Bottes; 27. Grotte sépulerale du Plan des Vaches; 28. Dolmen de la Blaque; 29. Stpulture des Vachons; 30. Station du Brugas; 31. Station da Verger). 32 a 34 Fragmentos donde el tipo de silex es indeterminado (O) (32. Dolmen du Chardonnet; 33. Dolmen des Plaines; 34. Grotte du Singla).

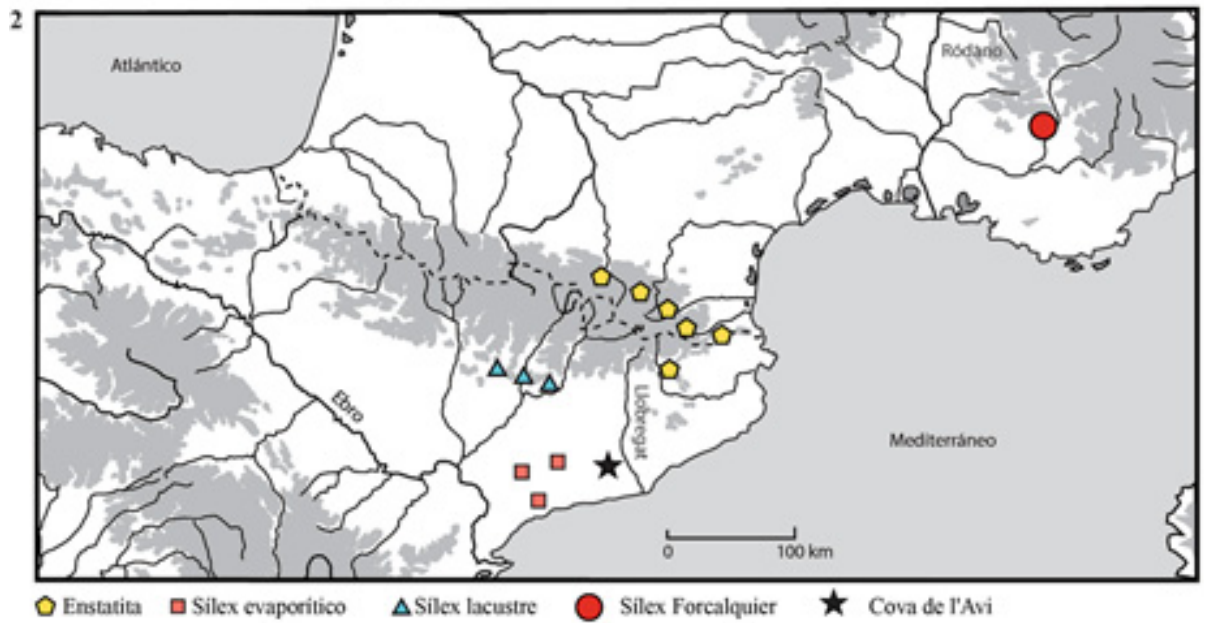

Fig. 5. 1. Mapa de distribución de los puñales de Châtaigniers y otros tipos. 2. Mapa de las posibles redes de intercambio en el Nordeste de la Península Ibérica durante el Neolítico final. La enstatita (Fig. 2.14) puede corresponder a esteatita pirenaica modificada por la acción del fuego. El sílex evaporítico, como el de las puntas de flecha (Fig. 3.1-3.5), es ampliamente conocido en la Depresión del Ebro, el lacustre (Fig. 3.6) con grandes carofíceas en los pre-Pirineos y el sílex Forcalquier (Fig. 3.9 y 3.10) en la Alta Provenza (dibujos J. Vaquer). 
sular. Pero en el Mediodía francés son propias del Neolítico final 2 (c. 3200-2800) y en el valle del Ebro presentan edades similares (Vegas 2007). En el Nordeste, sólo las puntas de perímetro serrado se asocian al Campaniforme. Los demás tipos se encuentran ya desde el Neolítico medio (Martín y Mestres et al. 2002; Juan Cabanilles 2008).

Finalmente, el puñal en bufanda de tipo Châtaigniers (Vaquer et al. 2014), tradicionalmente se relaciona con cronologías más recientes, concretamente con niveles calcolíticos del Mediodía Francés (Fig. 5). Sin embargo, solo en la localidad Hypogée des Crottes se dispone de una datación (4100 \pm 140 BP) para fechar el tipo (Sauzade 1983). En el Nordeste de la Península Ibérica, hay puñales similares en contextos parecidos al de la Cova de l'Avi (c. 4700 - 4500 BP), es decir, procedentes de excavaciones antiguas donde es difícil contextualizar los fósiles directores. El actual registro estratigráfico complica la resolución del desajuste cronológico de 700-1000 años entre los puñales documentados a uno y otro lado de los Pirineos.

Los tipos de punzones en hueso recuperados en la Cova de l'Avi son recurrentes durante toda la Prehistoria reciente de la Península Ibérica (Maicas 2007) y de Francia (Camps Fabrer 1990). Los punzones sobre asta de corzo son poco conocidos en el nordeste peninsular (Llobera 1986) y comunes en el grupo Saint-Pons del Neolítico final francés (Ambert 2003).

Los adornos de la Cova de l'Avi tienen analogías con los de otros grupos. El único paralelo del colgante bífido procede del Mediodía francés (Claustre y Guilaine 1981), aunque la fractura que afecta a la pieza peninsular impide conocer con exactitud si comparte idéntica forma. El colgante sobre diente se asocia en el sur de Francia al grupo Saint-Pons (Ambert 2003) y en el nordeste al contexto Veraza-Ferrières (Oms et al. 2010).

Las cuentas de collar sobre Cardium sp. corresponden a un tipo de adorno común con amplia dispersión cronocultural en el nordeste peninsular: desde localidades con el mismo marco cronológico a otras cercanas con ajuares del III y II milenio (Oliva 2006). También hay ejemplares regionales similares de las cuentas sobre mineral, aunque carecen de análisis físicos (Martín et al. 2002). En la Cova de l'Avi se ha podido identificar, por primera vez, la enstatita, un tipo de roca ultrabásica poco común en contextos sepulcrales franceses (Roscian et al. 1992) que aflora tanto en la pro- vincia de Girona como en las pegmatitas de los altos macizos del Ariège y en los Altos Pirineos (Roscian et al. 1992).

La analogía de ciertos materiales con el grupo Saint-Pons es difícil de definir por la ausencia de vasos cerámicos de dicha filiación. Por ello, no es descartable que los materiales líticos en sílex de Forcalquier, el punzón sobre asta, el colgante bífido en concha y el del diente de suido pudieran deberse a intercambios directos o indirectos. El grupo Saint-Pons se inicia c. 4700 BP (Ambert 2003) en un espacio limitado al norte del Languedoc occidental y representa la transición entre el Chassey y el Veraza.

\section{CONCLUSIONES}

Las dataciones de la Cova de l'Avi (c. 4500 $\mathrm{BP})$, junto con las de otros yacimientos del Nordeste de la Península Ibérica (Fig. 4), se sitúan entre las más antiguas de las cuevas sepulcrales, siendo coetáneas a las últimas sepulturas del Neolítico medio-reciente (Vallesiense). Además, nos indican que, a mediados del IV milenio cal BC, las redes de intercambio del Neolítico medio son substituidas por otras. Su mejor exponente sería el sílex de alta calidad de Apt-Forcalquier, fruto probable de la presencia de un artesanado especializado, que en el Mediodía francés tiene su máxima eclosión en el Calcolítico pleno (2800-2500), cuando los puñales de cobre aún son raros. Estos elementos circularían como objetos de prestigio, formando parte de algunos escasos enterramientos como marcadores de distinción de las élites. Valorar el porqué de este cambio tan profundo en las pautas de vida/muerte debe ser el objetivo de futuros trabajos.

\section{AGRADECIMIENTOS}

Este trabajo es parte del proyecto "El Plistocè Superior a la costa central catalana: paleoambients i ocupació dels neandertals" (2014/100639), financiado por el Servei d'Arqueologia i Paleontologia (Generalitat de Catalunya). La excavación de urgencia ha sido sufragada por el Servei de Arqueologia a través de la empresa ATICS y las

Trab. Prehist., 72, N. ${ }^{\circ}$ 2, julio-diciembre 2015, pp. 327-341, ISSN: 0082-5638

doi: $10.3989 /$ tp. 2015.12157 
analíticas mediante los proyectos 2014SGR-108, HAR2014-55131 y HAR2013-48010-P. J. Daura y M. Sanz han sido financiados con un contrato posdoctoral (SFRH/BPD/100507/2014 y SFRH/ BPD/100828/2014 respectivamente) de la Fundação para a Ciência e a Tecnologia (Portugal) con la financiación de FSE/POPH.

\section{BIBLIOGRAFÍA}

Ambert, P. 2003: "Défense et illustration du Néolithique récent du Languedoc central, plus particulièrement de celui de la région Saint-Pons-Lodève". Bulletin de la Société Préhistorique Française 100 (2): 357-374.

Asensio, A. 2001: "Recull de cavitats inèdites". Ardenya 1: 57-68.

Bosch, J. y Gómez, A. 2009: “Estudi de les ceràmiques neolítiques procedents de les mines 83, 84, 85 i 90 de Gavà. Intervencions arqueològiques a les Mines de Gavà (sector serra de les Ferreres). Anys 19982009". Rubricatum 4: 63-83.

Bronk Ramsey, 2013: OxCal 4.2.2 Manual. https://c14.arch.ox.ac.uk (consulta 6-V-2014).

Camps Fabrer H. y Ramseyer, D. 1990: "Fiche pointe sur andouiller de cervidé". En H. Camps Fabrer, D. Ramseyer y D. Stordeur (eds.): Fiches typologiques de l'industrie osseuse préhistorique, cahier III: poinçons, pointes, poignards, aiguilles. Publ. Université de Provence. Aix-en-Provence: 1-7.

Camps Fabrer, H.; Ramseyer, D. y Stordeur, D. 1990: Fiches typologiques de l'industrie osseuse préhistorique, cahier III: poinçons, pointes, poignards, aiguilles. Publ. Université de Provence. Aix-enProvence.

Cebrià, A.; Fullola, J. M.; López-Onaindia, D.; Mangado, X.; Nadal, J.; Ollé, A.; Oms, F. X.; Pedro, M.; Ruiz, J.; Subirà, M. E.; Torrente, A. y Vergès, J. M. 2013: "La cova sepulcral del pantà de Foix (Castellet i la Gornal). De jaciment arraconat a jaciment modèlic". En J. Hernández, J. Grau y J. Melero (ed.): III Monografies del Foix. Comunicacions de la III Trobada d'Estudiosos del Foix (Castellet $i$ la Gornal 2012). Documents de Treball. Sèrie Territori 25, Diputació de Barcelona. Barcelona: 184-194.

Claustre, F. y Guilaine, J. 1981: "La grotte de Bringairet, Armissan (Aude)". Actes du 106 Congrès national des Sociétés savantes (Perpignan 1981): 39-50. Paris.

Clua, M.; Daura, J. y Sanz, M. 2013: "El taller falsari de la Cova de l'Avi (Vallirana, Barcelona)". En A. Estrada-Rius (ed.): La falsificació de moneda a la Catalunya del segle XIX. Col-lecció d'Estudis d'Història del Dret 6, Universitat Pompeu Fabra. Barcelona: 141-164.
Gibaja, J. F. 2003: Comunidades neoliticas del Noreste de la Península Ibérica. Una aproximación socio-económica a partir del estudio de la función de los útiles líticos. British Archaeological Reports, International Series 1140, Archaeopress. Oxford.

Gibaja, J. F.; Majó, T.; Chambon, P.; Ruíz, J. y Subirà, M. E. 2010: "Prácticas funerarias durante el Neolítico. Los enterramientos infantiles en el noreste de la Península Ibérica". Complutum 21 (2): 47-68.

Gibaja, J. F.; González, P.; Martín, A.; Palomo, A.; Petit, M. A.; Plasencia, X.; Remolins, G. y Terradas, X. 2014: "New finds of obsidian blades at Neolithic sites in north-eastern Iberia". Antiquity Project Gallery, issue 340, june 2014. http://journal.antiquity. ac.uk/projgall/gibaja340 (consulta 10-VI-2014).

Gómez, A.; Tornero, C.; Saña, M. y Molist, M. 2011: "La Cova de les Agulles: un espai sepulcral entorn el III mil. cal. ane al Massís de l'Ordal". En A. Blasco, M. Edo y M. J. Villalba: La cova de can Sadurní i la prehistòria de Garraf. Recull de 30 anys d'investigació (Jornades Internacionals d'Arqueologia, Begues 2008). EDAR Arqueología y Patrimonio. Milán: 421-428.

Goodman, A. H. y Rose, J. C. 1990: "Assessment of Systemic Physiological perturbations from dental enamel hypoplasias and associated histological structures". Yearbook of Physical Anthropology 33: 59-110.

Juan Cabanilles, J. 2008: El utillaje de piedra tallada en la Prehistoria reciente valenciana. Aspectos tipológicos, estilísticos y evolutivos. Serie de Trabajos Varios del Servicio de Investigación Prehistórica 109. Valencia.

Llobera, X. 1986: "La Feixa del Moro (Juberri) $i$ el Neolític Mig-Recent a Andorra". Tribuna d'Arqueologia 1985-86: 14-24.

Maicas, R. 2007: Industria ósea y funcionalidad: Neolítico y Calcolítico en la Cuenca de Vera (Almería). Bibliotheca Praehistorica Hispana, CSIC. Madrid.

Martí, M.; Pou, R. y Carlús, X. 1997: La necròpolis del neolític mitjà $i$ les restes romanes de Can Grau (la Roca del Vallès, Vallès Oriental). Els jaciments de Cal Jardiner (Granollers, Vallès Oriental). Excavacions arqueològiques a Catalunya 14 , Generalitat de Catalunya. Barcelona.

Martín, A. 2003: "Els grups del neolític final, calcolític i bronze antic. Els inicis de la metal-lúrgia". Cota Zero 18: 76-105.

Martín, A. y Mestres, J. S. 2002: "Periodització des de la fi del neolític a l'edat del bronze a la Catalunya sud-pirinenca. Cronologia relativa i absoluta". XII Congrés Internacional de Puigcerdà: Pirineus $i$ veïns al $3^{r}$ mil·lenni $A C$. De la fi del neolitic a l'edat del bronze entre l'Ebre i la Garona (Puigcerdà 2000): 77-130. Puigcerdà.

Martín, A. y Villalba, M. J. 1999: “Le Néolithique moyen de la Catalogne". En J. Vaquer (ed.): XXI- 
VE Congrès Préhistorique de France (Carcassonne 1994). Le Néolithique du Nord-Ouest méditerranéen: 211-224. Paris.

Martín, A.; Petit, M. A. y Maya, J. L. 2002: “Cultura material, economia i intercanvis durant el III mil·lenni a Catalunya". XII Congrés Internacional de Puigcerdà: Pirineus $i$ veïns al $3^{r}$ mil-lenni $A C$. De la fi del neolitic a l'edat del bronze entre l'Ebre i la Garona (Puigcerdà 2000): 295-321. Puigcerdà.

Oliva, M. 2004: "Els ornaments personals de la primera meitat del segon mil·lenni ane del jaciment de Can Roqueta-II (Est), Sabadell". Cypsela 15: 229-249.

Oliva, M. 2006: "Els ornaments i les joies a la prehistòria de Sabadell". En Museu d'Art de Sabadell (ed.): Entre la intimitat i l'exhibició. La joia feta, portada $i$ venerada a Sabadell. Catàleg de l'exposició, 2 de maig - 2 de juliol 2006. Museu d'Art de Sabadell. Sabadell: 26-49.

Oliva, M. 2010: "Technology, production and use of malacology ornaments and tools in the prehistoric site of Can Roqueta (Barcelona, Spain)"'. En E. Álvarez y D. R. Carvajal (eds.): Not only food. Marine, terrestrial and freshwater molluscs in archaeological sites. $2^{\text {nd }}$ Meeting of ICAZ Archaeomalacology Working Group (Santander 2008). Munibe, Suplemento 31: 146-154.

Oms, F. X.; Pedro, M.; Bargalló, A.; López-García, J. M.; Morales, J. I. y Solé, À. 2010: "El projecte arqueològic a la serra del Montsec (Pallars Jussà). Noves dades per al coneixement del neolític i edat del bronze al Prepirineu de Lleida". En Grup de Prehistòria del Solsonès (ed.): $2^{n}$ Col $\cdot$ loqui d'Arqueologia d'Odèn (el Solsonès). Home i territori: darreres investigacions al Prepirineu lleidatà 2006-2008 (Odèn 2009): 39-46. Odèn.

Panei, L.: Rinaldi, G. y Tosi, M. 2005: "Investigations on ancient beads from the Sultanate of Oman (Ra's al-Hadd-Southem Oman)". Archéo Sciences, Revue d'Archéometrie 29: 151-155.

Petit, M. A. 2001: "Els primers pagesos i ramaders. De la $2^{\text {a }}$ meitat del VI mil·lenni a mitjan del III ${ }^{\text {er }}$ mil·lenni cal. BC". En J. Giralt (ed.): La Noguera antiga. Des dels primers pobladors fins als visigots. Museu de la Noguera-Museu d'Arqueologia de Catalunya. Girona: 46-61.

Petit, M. A. y López-Cachero, F. X. 2011: "De la fi del Neolític a l'Edat del Bronze al Garraf-Ordal". En A. Blasco, M. Edo y M. J. Villalba: La cova de can Sadurní i la prehistòria de Garraf. Recull de 30 anys d'investigació (Jornades Internacionals d'Arqueologia, Begues 2008). EDAR Arqueología y Patrimonio. Milán: 367-387.

Reimer, P. J.; Bard, E.; Bayliss, A.; Beck, J. W.; Blackwell, P. G.; Bronk Ramsey, C.; Buck, C. E.; Cheng, H.; Edwards, R. L.; Friedrich, M.; Grootes, P. M.; Guilderson, T. P.; Haflidason, H.; Hajdas, I.; Hatté, C.; Heaton, T. J.; Hoffmann, D. L.; Hogg, A. G.;
Hughen, K. A.; Kaiser, K. F.; Kromer, B.; Manning, S. W.; Niu, M.; Reimer, R. W.; Richards, D. A.; Scott, E. M.; Southon, J. R.; Staff, R. A.; Turney, C. S. M. y van der Plicht, J. 2013: "IntCal13 and Marine13 radiocarbon age calibration curves $0-50,000$ years cal BP”. Radiocarbon 55 (4): 1869-1887.

Renault, S. y Bressy, C. 2007: "Les recherches en contexte d'atelier depuis la fin du $\mathrm{XIX}^{\mathrm{e}}$ siècle en Provence: de la recherche des artefacts à l'approche pluridisciplinaire". En J. Évin (ed.): Un siècle de construction du discours scientifique en Préhistoire. Actes du XXVI Congrès Préhistorique de France, Congrès du centenaire de la Société Préhistorique Française (Avignon 2004) II: 279-295. Paris.

Roig, J.; Coll, J. M.; Gibaja, J. F.; Chambon, Ph.; Villar, V.; Ruiz, J.; Terradas, X. y Subirà, M. E. 2010: "La Necrópolis de Can Gambús-1 (Sabadell, Barcelona). Nuevos conocimientos sobre prácticas funerarias durante el Neolítico medio en el Noreste de la Península Ibérica". Trabajos de Prehistoria 67 (1): 59-84.

Roscian S.; Claustre F. y Dietrich J.-E. 1992: "Les parures du Midi méditerranéen du Néolithique ancien à l'Age du Bronze: origine et circulation des matières premières". Gallia Préhistoire 34: 209-257.

Sauzade, G. 1983: Les sépultures du Vaucluse du néolithique à l'Age du Bronze. Col. Études Quaternaires 6, Éditions de l'Université de Provence, Institut de Paléontologie Humaine. Paris.

Subirà, M. E.; Lopez-Onaindia, D. y Yll, R. 2014: “Cultural changes in funeral rites during the Neolithic in the Northeast of the Iberian Peninsula? The cave of Pantà de Foix (Barcelona)". International Journal of Osteoarchaeology. DOI: 10.1002/oa. 2400.

Terradas, X.; Gratuze, B.; Bosch, J.; Enrich, R.; Esteve, X.; Oms, F. X. y Ribé, G. 2014: "Neolithic diffusion of obsidian in the western Mediterranean: new data from Iberia". Journal of Archaeological Science 41: 69-78.

Vaquer, J. 2012: "Réflexions sur échanges de biens matériels lithiques entre le midi de la France et le nord de la péninsule Ibérique au néolithique et au chalcolithique". En M. Borrell, F. Borrell, J. Bosch, X. Clop y M. Molist: Actes del Congrés Internacional Xarxes al Neolític - Neolithic Network (GavàBellaterra 2011). Rubricatum 5: 565-574.

Vaquer, J.; Martín, A.; Pétrequin, P.; Pétrequin, A. M. y Errera, M. 2012: "Les haches alpines dans les sépultures du Néolithique moyen pyrénéen: importations". En P. Pétrequin, S. Cassen, M. Errera, L. Klassen, A. Sheridan y A. -M. Pétrequin (eds.): Jade. Grandes haches alpines du Néolithique européen. $V^{E}$ et $I V^{E}$ millénaires av. J.-C. Presses Universitaires de Franche-Comté 1224. Besançon: 872-917.

Vaquer, J.; Martín, A.; Juan-Cabanilles, J.; Bordreuil, M. y Galant, P. 2014: "Les poignards à retouches parallèles couvrantes sur préformes polies en silex de Forcalquier dans la zone nord-occidentale de la 
Méditerranée". En R.-M. Arbogast y A. GreffierRichard (ed.): Entre archéologie et écologie, une préhistoire de tous les milieux. Mélanges offerts à Pierre Pétrequin. Presses Universitaires de Franche-Comté, Annales Littéraires de l'Université de Franche-Comté 928, série Environnement, Sociétés et Archéologie 18. Besançon: 129-155.

Vegas, J. I. 2007: San Juan Ante Portam Latinam. Una inhumación colectiva prehistórica en el valle medio del Ebro. Memoria de las excavaciones arqueológi- cas, 1985, 1990 y 1991. Diputación Foral de Álava, Dept. Cultura y Euskera. Vitoria-Gasteiz.

Villalba, M. J.; Edo, M. y Blasco, A. 2011: "Les mines neolítiques de Can Tintorer. Una relectura trenta anys després". En A. Blasco, M. Edo y M. J. Villalba: La cova de can Sadurní $i$ la prehistòria de Garraf. Recull de 30 anys d'investigació (Jornades Internacionals d'Arqueologia, Begues 2008). EDAR Arqueología y Patrimonio. Milán: 293-335. 